\title{
Mechanistic Study of Defect-Induced Oxidation of Graphite
}

\author{
J. R. Hahn and H. Kang* \\ Department of Chemistry and Center for Ion-Surface Reaction, Pohang University of Science and Technology, \\ Pohang, Gyeong-buk, 790-784, South Korea
}

\begin{abstract}
S. M. Lee and Y. H. Lee
Department of Semiconductor Science and Technology, Semiconductor Physics Research Center, Jeonbuk National University, Jeonju, 561-756, South Korea
\end{abstract}

Received: June 23, 1999; In Final Form: September 5, 1999

\begin{abstract}
Oxidation of a graphite initiated from surface defects is investigated by using scanning tunneling microscopy (STM) and density functional (DF) calculation. The defects are created on a graphite surface with controlled depth and density by impact of $\mathrm{Ar}^{+}$ions at low energies $(50-500 \mathrm{eV})$. Oxidation of the defects with $\mathrm{O}_{2}$ at temperatures of $450-650{ }^{\circ} \mathrm{C}$ produces shallow pits on the surface via layer-by-layer etching of the carbon layer. The etch pattern and speed vary depending on the depth of a pit. Round shaped pits are produced in most cases, but less frequently anisotropic patterns are also observed for monolayer etching. A multilayer pit grows a few times faster than a monolayer pit via simultaneous etching of the top and inner carbon layers. Kinetic parameters are extracted for the oxidation of depth-differentiated pits. The activation energies are quite comparable for the oxidation of monolayer and multilayer pits, but the pre-exponential factor is larger for multilayer oxidation, which gives rise to a faster etching speed. DF calculations are performed to identify the key intermediates involved in the oxidation reactions. The calculation shows that an $\mathrm{O}_{2}$ molecule dissociates exothermally with no energy barrier and then forms oxide species at the top or bridge sites of a carbon vacancy. A metastable $\mathrm{O}_{2} *$ precursor state, in which an $\mathrm{O}_{2}$ molecule adsorbs at the bridge site without dissociation, is also found. These surface species can be desorbed under the etching conditions either thermally or via further chemical reactions. Reaction pathways involving these intermediates are proposed to explain the different etching behaviors observed for mono- and multilayer pits.
\end{abstract}

\section{Introduction}

Thermal oxidation of a graphite surface is technologically an important subject, applicable to the industrial processes such as coal gasification, combustion, water-gas production, and the researches for nuclear reactor materials and reentry shields of space crafts. The reaction also provides a good model system for scientific understanding of gas-solid reactions because of the low dimensionality of a graphite lattice and the simplicity in kinetic simulation involving only a few oxidation products.

Transmission electron microscopy (TEM) and an etch-gold decoration technique incorporated to TEM (ED-TEM) have been widely employed in early studies of graphite oxidation. ${ }^{1-14}$ Several excellent reviews have been given on this subject. ${ }^{1-4}$ In these works, thermal oxidation of graphite led to formation of shallow pits in the basal plane. The pits were believed to originate from the natural point defects present on a surface, and the depth of pits was assumed to be a graphite monolayer. Two types of mechanisms were proposed for the reaction of $\mathrm{O}_{2}$ molecules with graphite defects: ${ }^{10,11}$ (i) reaction initiated by direct interaction of gas-phase $\mathrm{O}_{2}$ molecules with defect sites [Eley-Rideal (ER) mechanism]; (ii) reaction involving surface migration of oxygen, or the Langmuir-Hinshelwood (LH) mechanism. In the $\mathrm{LH}$ mechanism, $\mathrm{O}_{2}$ is first physisorbed onto a basal plane and then diffuses to encounter the active sites of the surface. It is uncertain, though, what is the bonding character

* Corresponding author. E-mail: surfion @ postech.ac.kr. Fax: +82-562279-8157. of the diffusive oxygen species on the surface. Thermal oxidation of graphite step edges and their etch kinetics have also been studied. 5,6,15 The etch rate reported from these works showed considerably scattered numbers depending on the reaction temperatures and whether the etching involved monolayer or multilayer steps. Since TEM or ED-TEM techniques were used in these works, accurate measurement of the step heights was difficult. STM was also employed for examination of graphite oxidation. ${ }^{15-20}$ STM revealed that only monolayer pits were formed by oxidation of natural defects. It was found ${ }^{15-19}$ that $\mathrm{O}_{2}$ molecules did not induce vertical etching of a basal plane at temperatures below $650{ }^{\circ} \mathrm{C}$. Despite these discoveries, mechanistic details for the defect-induced oxidation of graphite are still not well understood. Such difficulty arises mainly from two reasons: (i) the nature of graphite defects was poorly identified in previous works, due to experimental difficulty of characterizing the natural defects at low density $\left(0.1-15 \mu \mathrm{m}^{-2}\right)$; (ii) an appropriate molecular model was absent for the reaction of $\mathrm{O}_{2}$ molecules with graphite defects, which is an essential ingredient for understanding the oxidation mechanism.

Recently, microwave Ar plasma ${ }^{17}$ or low-energy $(<500 \mathrm{eV})$ ion beams ${ }^{22-29}$ have been used to generate defects on a graphite surface and to have control of their property. Such efforts are desirable for future nanoscience applications such as markers and molecular corrals. ${ }^{21}$ The types of defects (vacancy and interstitial) were able to be identified and distinguished by applying scanning tunneling spectroscopy (STS) to the defects 
created by low-energy ions. ${ }^{24}$ The defect density and depth were controlled by using low-energy ions at selected dose and incident energy. ${ }^{24-26}$ Oxidative etching was observed separately from vacancy and interstitial, ${ }^{27}$ and it was found that mainly the vacancies were converted to pits by oxidation, while the interstitials exhibited a relatively low conversion efficiency.

In the present paper, we report a mechanistic study for the oxidative etching of ion-induced defects on graphite. Our initial observation of the defect oxidation and its mechanistic interpretation has recently been reported. ${ }^{28}$ This work is distinguished from the previous studies ${ }^{5-20}$ in several aspects. The defects are intentionally generated on a surface with controlled depth and density by energy-selected $\mathrm{Ar}^{+}$ion impact. The oxidation reaction is examined for thus-created defects in a depthdifferentiated manner. Such an approach allows us to examine the oxidation behaviors as a function of defect depth, viz., the geometrical pattern of defect etching, the etch rate, the activation energy, and the entropy factor. These experimental observations are interpreted based on the molecular reaction model provided by DF calculations.

\section{Experimental Section}

Highly oriented pyrolytic graphite (Advanced Ceramics, ZYA grade) samples were cleaved with an adhesive tape in air and then loaded into the ion-surface collision chamber through a sample transfer system. ${ }^{30}$ The ion-surface collision chamber ${ }^{31}$ consisted of an ion source, a beam transport region, a low-energy decelerator, and an ultrahigh vacuum (UHV) chamber equipped with various surface analysis tools. The base pressure of the chamber was $2 \times 10^{-10}$ Torr. The sample was bombarded with mass- and energy-selected $\mathrm{Ar}^{+}$ion beams in the energy range of $50-500 \mathrm{eV}$. The defect population was controlled by the ion dose to have a surface density between $1 \times 10^{11}$ and $1 \times$ $10^{13} \mathrm{~cm}^{-2}$. Such defect density was much higher than a natural defect density $\left(10^{8} \mathrm{~cm}^{-2}\right)$, but still the defect-defect interactions were negligible on the surface. After ion impact, the sample was transferred to air for subsequent STM measurement and thermal oxidation.

Thermal oxidation of the ion-bombarded graphite was carried out in a furnace oven. A sample was placed on a clean alumina boat, and then loaded into the furnace. The sample was oxidized by heating it in dry air at temperatures between 450 and 650 ${ }^{\circ} \mathrm{C}$. These temperatures were lower than the ones in the previous studies, ${ }^{5-20}$ because the etch rate needed to be slowed on the present surface with a higher defect density. The oxidation temperature was monitored at the boat by attaching thermocouple wires. The time for oxidation ranged from several minutes to several tens of minutes. After oxidation, the sample was taken out and then cooled quickly by a flow of fresh air. We used a homemade STM to investigate the sample surfaces. The STM instrumentation has been described elsewhere. ${ }^{26}$

\section{Experimental Results}

3.1. Layer-by-Layer Oxidative Etching of Defect. STM images of Figure 1 show evolution of an ion-impacted graphite surface during thermal oxidative etching. In Figure 1a, the small bright spots represent the surface defects before oxidation, generated by $\mathrm{Ar}^{+}$ion impact at an energy of $150 \mathrm{eV}$. The bright spots, or surface protrusions, consist of both graphite vacancies and interstitials, the latter formed by trapping incident Ar atoms underneath the surface plane. ${ }^{24,26}$ When this surface is oxidized by $\mathrm{O}_{2}$ gas at $560{ }^{\circ} \mathrm{C}$, the bright spots are developed into nanometer-sized pits via lateral etching (Figure 1b). The pits with two different sizes are found on this surface, the ones with a smaller diameter correspond to monolayer pits, and the larger ones, which appear darker in the STM image, are double-layer pits. The cross-sectional cut of the image (Figure 1f) is provided to show the two different depths (3.35 and $6.70 \AA$ ). The relative population of the monolayer and double-layer pits varies with ion impact energy. Ions with higher energy produce a relatively larger portion of double-layer pit, and this behavior shows correlation with an increased population of the double-layer defects formed at higher energy. Monolayer pits are of a very uniform size, and so are double-layer pits. This indicates that the growth rate is almost the same for the pits of identical depth and also that the etching reactions start simultaneously from all defects upon sample heating. ${ }^{15}$ The graphite lattice pattern is visible in a high-resolution STM image at a terrace region, both inside and outside of a pit, but it is difficult to obtain at pit boundaries.

An interesting feature for the growth of double-layer pits is that the first and second carbon layers are etched away simultaneously (Figure 1f). Such simultaneous etching of multiple carbon layers occurs also for triple- and quadruplelayer pits (not shown). Vertical etching does not take place at an undamaged graphite plane by reaction with $\mathrm{O}_{2}$ at temperatures below $650{ }^{\circ} \mathrm{C} . .^{15-19}$ Therefore, the multilayer pits must have originated from the ion-generated defects of corresponding depths, by the simultaneous etching process.

As the oxidation time is further increased, the pits grow to result in overlap between their boundaries (Figure 1c). The overlap between the monolayer and double-layer boundaries gives rise to interesting phenomena. The fast etch rate of a double-layer pit becomes substantially retarded at the colliding boundary, as the simultaneous double-layer etching process is prohibited. A double-layer pit at this stage cannot maintain its circular shape any further. Upon prolonged oxidation (Figure 1d), the layer-by-layer etching process removes most of the first carbon layer, and the underlying second carbon layer becomes surfaced as a new terrace region. On this new surface, the original double-layer pits, now having a monolayer depth, behave much like monolayer pits in their etch speed and pattern. Eventually, the second carbon layer becomes all etched away, and the etching process continues for the deeper layers.

The etching produces circular shaped pits in most cases, but for monolayer etching, anisotropic patterns are also observed with less frequency. The anisotropic etching often results in a snakelike or serpentine structure. Figure 1e exemplifies such a feature, and the similar structures can also be seen in parts $b$ and $\mathrm{d}$ of Figure 1. The anisotropic etching does not occur for multilayer pits. Various types of etching patterns have been reported $^{13,14,16}$ in the presence of alkali metals or other reagents on a graphite surface. To check the effect of alkali metal adsorbates, we have examined the etching pattern after depositing $\mathrm{Cs}$ on the surface and found that the occurrence and size of the serpentine structures were not significantly affected by the presence of Cs. Therefore, the anisotropic etching appears to be an intrinsic characteristics of graphite monolayer etching, in addition to the major production of circular pits. However, we cannot completely rule out the possibility that the graphite samples were contaminated from a heated alumina boat.

3.2. Etch Rate versus Pit Depth. Figure 2a plots the average diameter of etched pits as a function of oxidation time. A linear increase of the diameter with time is observed quite consistently for the pits of all depths. The etch rate is smallest for monolayer pits $\left(1.38 \mathrm{~nm} / \mathrm{min}\right.$ at $\left.560{ }^{\circ} \mathrm{C}\right)$, and increases by $2-3$ times as the pit depth increases by one carbon layer up to three (Figure $2 b)$. For triple- and quadruple-layer pits, their etch rates are 

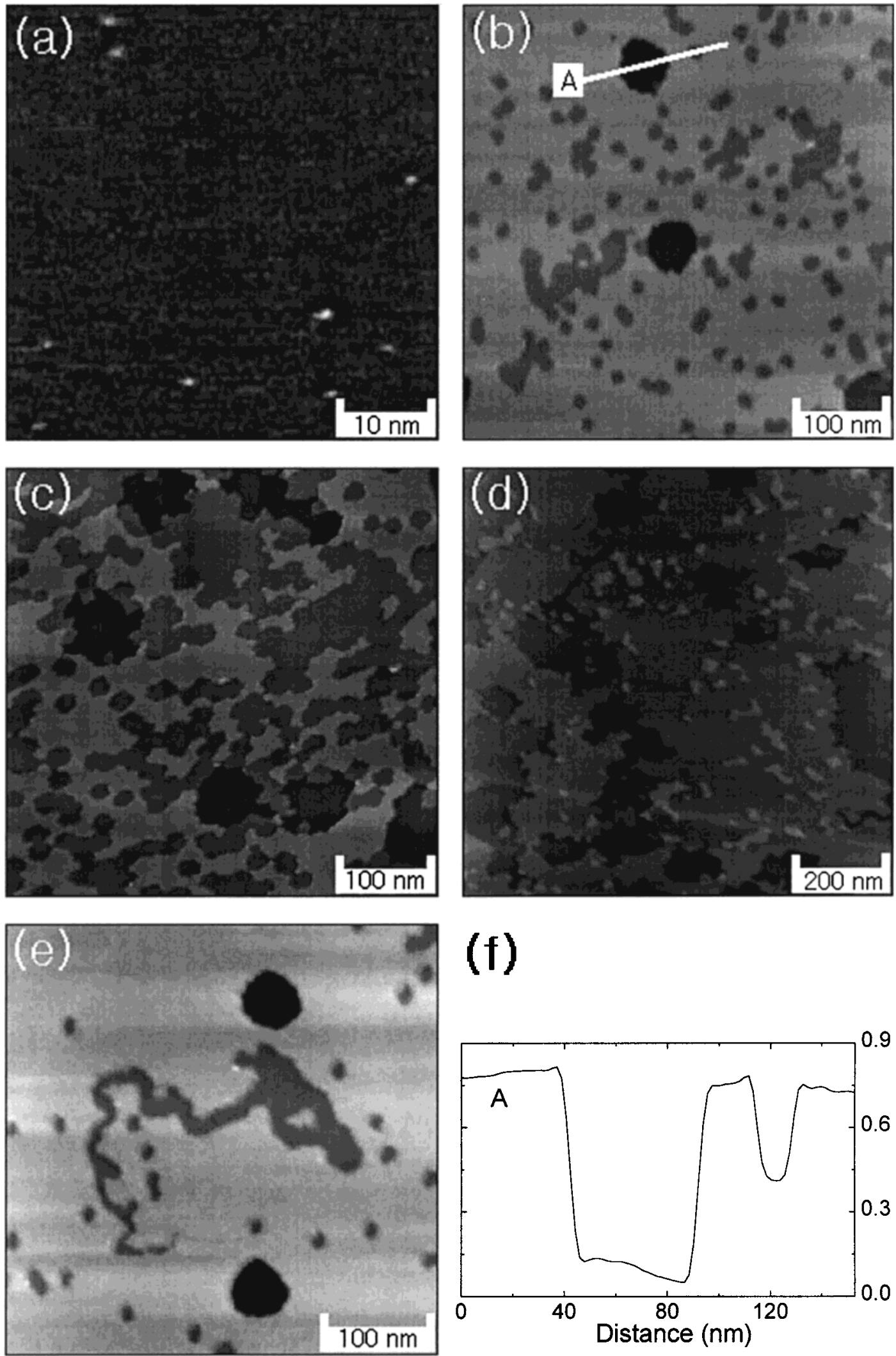

\section{$(f)$}

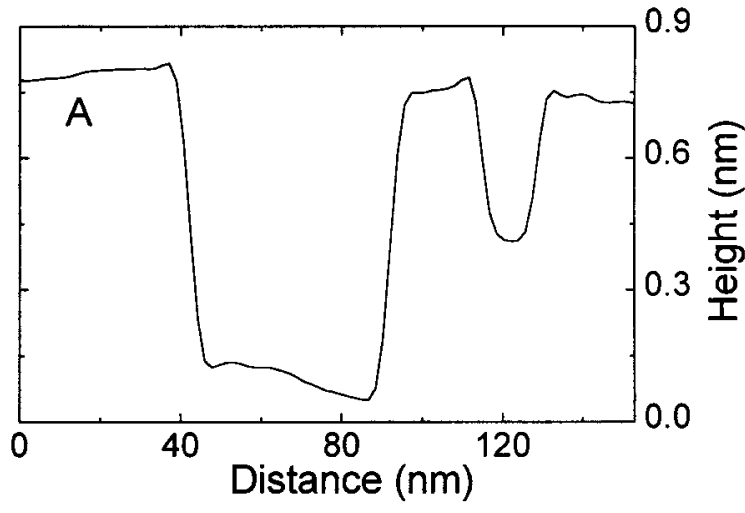

Figure 1. (a) Topographic STM image of a graphite surface bombarded with $150 \mathrm{eV} \mathrm{Ar}^{+}$ions. The bright spots represent the point defects. (b) Pits are formed from the defects upon heating surface a at $560^{\circ} \mathrm{C}$ for $17 \mathrm{~min}$. Double-layer pits have a larger diameter than monolayer pits. (c) The size of the pits increases after additional heating of surface b for $5 \mathrm{~min}$. (d) Most of the top carbon layer is removed after heating surface c for additional 10 min. (e) An anisotropic etch pattern observed for monolayer etching. (f) The cross-sectional cut along line A in surface b. All STM images are taken from one sample but from different surface regions. The numbers inside the white bar correspond to the bar lengths.

similar. With $\mathrm{Ar}^{+}$ion energy smaller than $500 \mathrm{eV}$, no pits are formed deeper than four layers. Extrapolation of the linear fits to the data of Figure 2a gives negative intercepts at time zero. It cannot be judged whether the negative intercept is caused by the inherent initial retention time in defect oxidation or by the delayed heating of the samples inside an oven.

Figure 3 illustrates another example of depth-dependent etch rate, but this time demonstrated for line steps. The STM image 

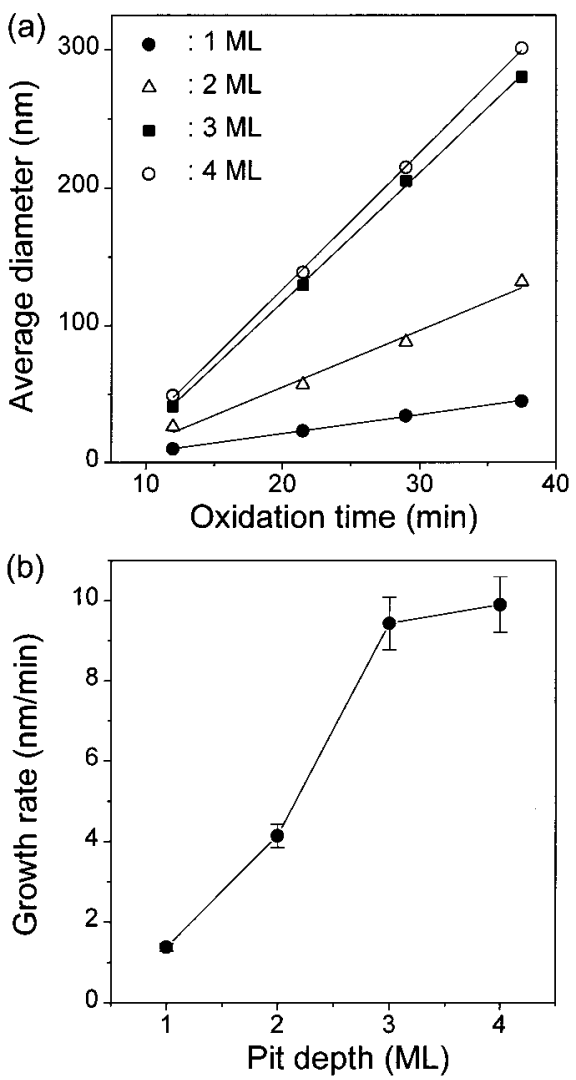

Figure 2. (a) The increase of average pit diameters versus oxidation time. The data are shown for single-, double-, triple-, and quadruplelayer pits. The lines are linearly fitted. Reaction temperature is 560 ${ }^{\circ} \mathrm{C}$. (b) Variation of the average growth rate with pit depth.

contains two line steps, the one in the left-hand side has a double-layer height and the other in the right a monolayer height. The randomly distributed pits originate from etching of the defects produced by $100 \mathrm{eV} \mathrm{Ar}^{+}$ions. At this energy, all the pits have monolayer depth. It is noticed that no pits exist in the terrace region near the double-layer step (the region between the double step and the white arrows). Apparently, the doublestep edge has been receded by about this distance by the etching, and the monolayer pits in this region have been washed away. On the other hand, several monolayer pits are found close to the monolayer line step. This indicates that the receded distance of the monolayer step is much smaller, comparable to the size of a monolayer pit. These features show another evidence for the higher etch rate at double-layer carbon edges.

3.3. Temperature Effect on the Etch Rate. The etch rate is measured as a function of oxidation temperatures between 500 and $650{ }^{\circ} \mathrm{C}$ for depth-differentiated pits. The size of a pit increases linearly with oxidation time at these temperatures as previously shown in Figure 2, and the slope of a linear increase gives an etch rate. The rate increases substantially with temperature, in agreement with the previous results. ${ }^{16,18}$ For the purpose of obtaining a quantitative temperature dependency of etch rate, the rate constant is defined by the turnover frequency, $r,{ }^{11}$ where $r$ represents the number of carbon atoms removed per unit time per active carbon site (dangling bond), as expressed by eq 1 . The temperature dependence of $r$ is assumed to follow an Arrhenius type (eq 2).

$$
\begin{aligned}
r\left(\mathrm{~s}^{-1}\right) & =n \rho_{0001}(2 \pi D)(\mathrm{d} D / \mathrm{d} t) / \rho_{1120}(2 \pi D) n h \\
& =A \exp \left(-E_{\mathrm{a}} / R T\right)
\end{aligned}
$$

In eq $1, \rho_{0001}$ is the areal density of carbon atoms at the (0001)
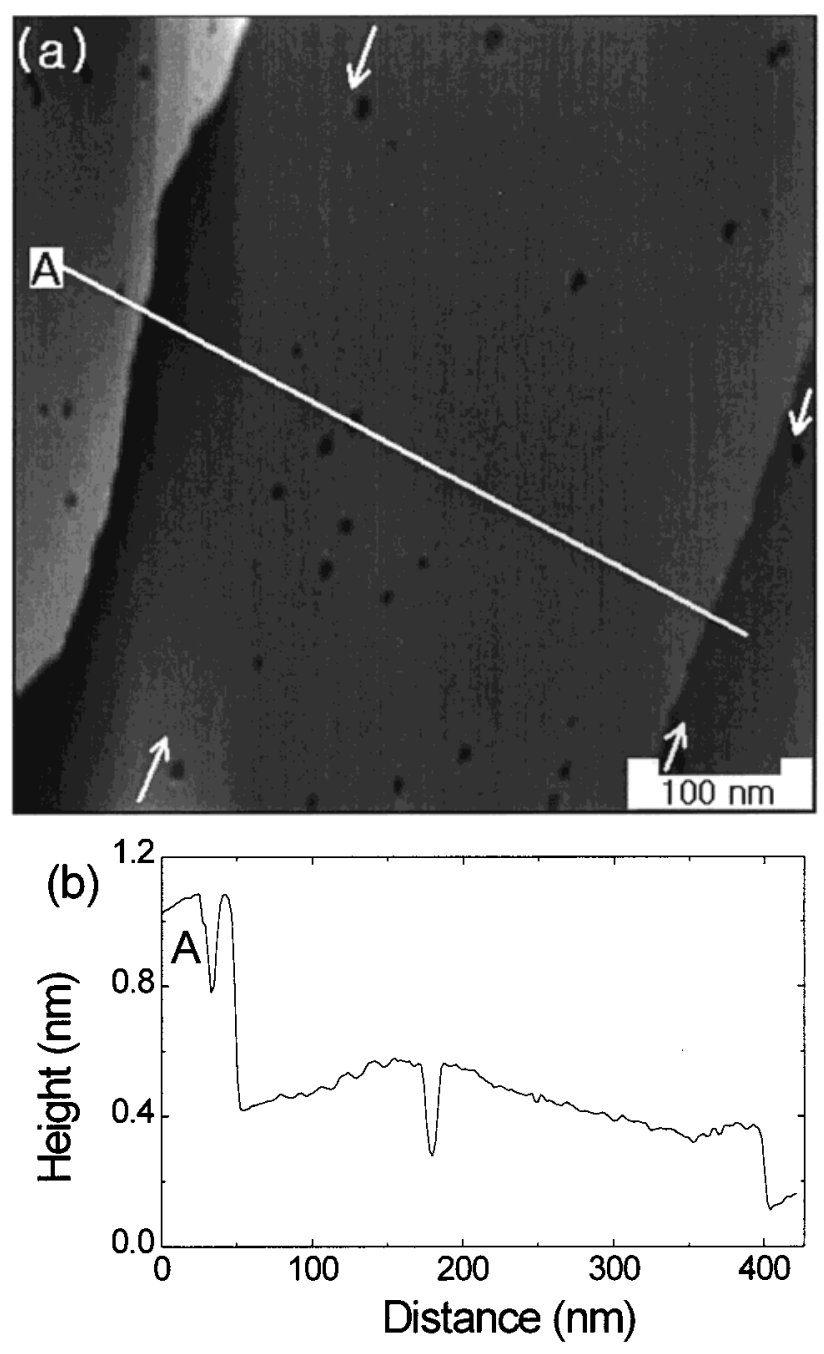

Figure 3. (a) STM image showing the etching behaviors of line step edges. A double-layer step is present in the left-hand side and a monolayer step in the right-hand side. The white arrows mark the assumed initial positions of the step edges before etching. The round pits are generated from ion-induced defects. (b) The cross-sectional cut along line A.

basal plane $\left(0.377\right.$ atoms $\left./ \AA^{2}\right), \rho_{1120}$ is the areal density of active carbon sites at the (1120) facet $\left(0.120\right.$ sites $\left./ \AA^{2}\right), n$ is an index for pit depth (1 for monolayer, 2 for double-layer, and so on), $h$ is the monolayer height, and $D$ is a pit diameter. In eq 2, $A$ is the pre-exponential factor of the Arrhenius formula and $E_{\mathrm{a}}$ is the activation energy. Figure 4 shows the Arrhenius plot of $r$ for single-, double-, and triple-layer pits.

From the fitting of Figure 4, the activation energy barriers are obtained for pit etching: $E_{\mathrm{a}}=96 \pm 8 \mathrm{~kJ} / \mathrm{mol}$ (monolayer), $109 \pm 5 \mathrm{~kJ} / \mathrm{mol}$ (double-layer), and about $130 \mathrm{~kJ} / \mathrm{mol}$ (triplelayer). The uncertainty in the triple-layer value must be larger because only two data points are available. The corresponding pre-exponential factors are $10^{4}-10^{5} \mathrm{~s}^{-1}$ (monolayer), $10^{6} \mathrm{~s}^{-1}$ (double-layer), and $10^{8} \mathrm{~s}^{-1}$ (triple-layer). An interesting feature of this analysis is that the $E_{\mathrm{a}}$ values are similar for the singleand double-layer etching within the error limits. However, the $A$ factor is larger for a double-layer pit, and this gives rise to the higher etch rate. The different $A$ values for mono- and multilayer etching suggest the importance of the entropy effect in the etching process.

\section{Theoretical Calculation}

4.1. Calculational Methods. We have performd DF total energy calculations based on local density approximation (LDA) 


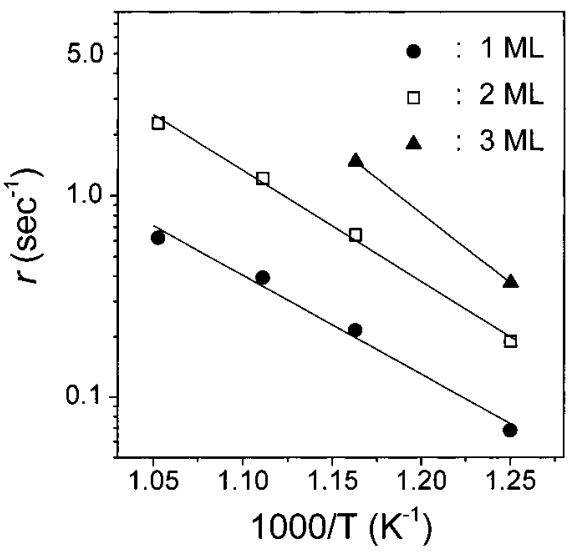

Figure 4. Arrhenius plots for the temperature dependence of etch rate constant $(r)$ presented for single-, double-, and triple-layer pits. The lines are least-squares linear fitting.

and generalized gradient approximation (GGA), as implemented in the Dmol3 code. ${ }^{32}$ The exchange-correlation energy in LDA is parametrized by Perdew and Wang's scheme ${ }^{33}$ and Becke's corrected exchange functional ${ }^{34}$ is adopted in GGA calculations. All-electron Kohn-Sham wave functions are expanded in a local atomic orbital basis. All orbitals including core electrons are taken into account throughout the calculations. In the doublenumerical basis set, $\mathrm{C}-2 \mathrm{~s}$ and $\mathrm{C}-2 \mathrm{p}$ orbitals are represented by two basis functions each, and a 3d-type wave function on carbon and oxygen is used to describe polarization. The convergency criterion for the structure optimization is that all forces on atoms be within 0.002 atomic units $(\mathrm{au})$ and the energy change for the charge density per simulation step be within $1.0 \times 10^{-5} \mathrm{au}$. Structure optimization is done by the LDA scheme only. The GGA calculations are done with the structures optimized by LDA whenever necessary.

A graphite surface is modeled by introducing a supercell of a $(4 \times 4)$ graphitic sheet with periodic boundary conditions applied along the planar directions to simulate an infinite graphitic layer. The distance between layers is $3.4 \AA$, and we neglect van der Waals interaction between layers, since this interaction is much weaker than the covalent one. The surface is simulated by periodically repeated slabs of a graphitic monolayer parallel to the surface direction with a vacuum region of $7 \AA$. A carbon atom is removed to create a vacancy, and the whole structure is fully relaxed by the steepest descent optimization scheme within the LDA method. The binding energy of graphite $(-820 \mathrm{~kJ} / \mathrm{mol}$ of carbon) is overestimated compared with the experimental value $(-710 \mathrm{~kJ} / \mathrm{mol})$, as expected from LDA calculations. However, we expect the energy difference of different structures to be of a small error. We have tested the size dependence using a larger size $(6 \times 6)$ cell. Changes in energies were within $10 \mathrm{~kJ} / \mathrm{mol}$ of carbon. We have used Fermi-level smearing of 0.1 au for fast convergence in the geometry optimization. The absolute binding energy became weaker, but changes in relative energies were negligible.

4.2. Oxidation of Monolayer Carbon Vacancy. Single monovacancy is created at the top carbon layer and the whole structure is fully relaxed. Carbon atoms with dangling bonds at the monovacancy form short bonds $(1.38 \AA$ ) with their adjacent carbon atoms, compared to an ideal bond of graphite $(1.42 \AA)$. We now add an $\mathrm{O}_{2}$ molecule on this vacancy. Although we have tried various initial configurations, ${ }^{28}$ an $\mathrm{O}_{2}$ molecule exothermally dissociates and $\mathrm{O}$ atoms chemisorb on the top and bridge sites in all cases, as shown in Figure 5a. The exothermic energy for creating two oxides at the top and bridge sites from an $\mathrm{O}_{2}$ molecule is $936(859) \mathrm{kJ} / \mathrm{mol}$ from LDA (GGA) (a)

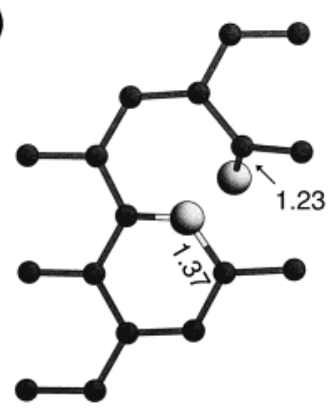

(c)

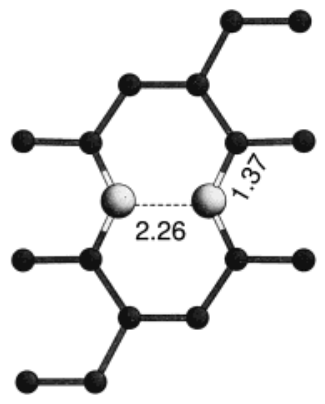

(b)

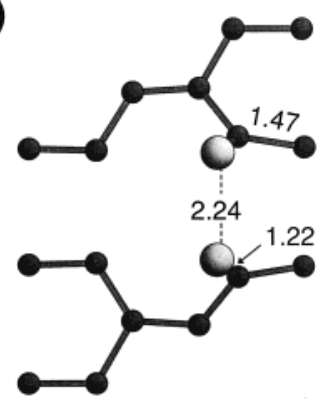

(d)

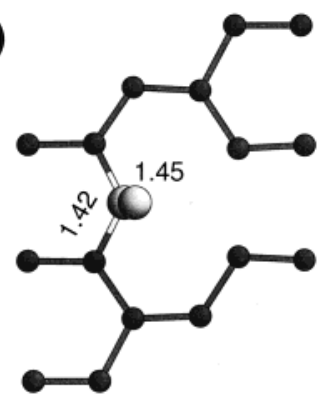

\section{: Carbon $\quad$ : Oxygen}

Figure 5. Fully relaxed local geometries in a ball-and-stick form for $\mathrm{O}_{2}$ adsorption at vacancies on single graphitic layer. (a) $\mathrm{O}_{2}$ dissociative adsorption at a monovacancy, (b) dissociative adsorption at two top sites of a divacancy, where two oxygen atoms are located above the graphitic plane, (c) dissociative adsorption at two bridge sites of a divacancy, and (d) an $\mathrm{O}_{2}$ molecularly adsorbed at a single bridge site. Bond lengths are in units of angstroms.

calculations. No barrier exists for this chemisorption. When an $\mathrm{O}_{2}$ molecule approaches the dangling bonds at a vacancy, charges are transferred from carbon atoms to the oxygen molecule due to the larger electronegativity of oxygen atoms. This interaction keeps the oxygen atoms apart, placing them at the opposite sides of the plane as shown in Figure 5a. The resulting intramolecular Coulomb repulsion breaks the $\mathrm{O}-\mathrm{O}$ bond exothermally without an activation barrier.

We calculate the adsorption of an $\mathrm{O}_{2}$ molecule at a divacancy. Structures are relaxed fully similar to the case of a monovacancy. One of the back-bonds is contracted to $1.38 \AA$. When an $\mathrm{O}_{2}$ molecule approaches to the middle of two dangling bonds at a divacancy with the $\mathrm{O}_{2}$ molecular axis parallel to the atomic plane, it exothermally dissociates and chemisorbs at two top sites as shown in Figure 5b. The exothermic energy of forming two $\mathrm{C}-\mathrm{O}$ bonds at the top sites from an $\mathrm{O}_{2}$ molecule is 1158 (1003) kJ/mol from LDA (GGA) calculations (strictly speaking, this includes the relaxation energy of adjacent carbon atoms). The $\mathrm{C}-\mathrm{O}$ bond length is $1.22 \AA$, almost the same as $1.23 \AA$ of a $\mathrm{C}-\mathrm{O}$ bond of the top site at a monovacancy, but a little longer than that of a free $\mathrm{CO}$ molecule $(1.13 \AA)$. The $\mathrm{O}-\mathrm{O}$ distance is $2.24 \AA$, with oxygen atoms repelling each other. The situation is different when an $\mathrm{O}_{2}$ molecule adsorbs at the center of a divacancy parallel to the missing carbon dimer. $\mathrm{An}_{2}$ molecule now saturates all the dangling bonds, by taking two bridge sites as shown in Figure 5c. The exothermic energy of forming two $\mathrm{C}-\mathrm{O}$ bonds at the bridge site is $1602(1370) \mathrm{kJ} / \mathrm{mol}$ from LDA (GGA) calculations. The $\mathrm{C}-\mathrm{O}$ bond length is $1.37 \AA$, the same as that at the bridge site of a monovacancy. The $\mathrm{O}-\mathrm{O}$ distance is $2.26 \AA$, similar to that at two top sites. We find another stable configuration, an $\mathrm{O}_{2} *$ molecular precursor state, as shown in Figure $5 \mathrm{~d}$. This occurs when an $\mathrm{O}_{2}$ molecule approaches the 
bridge site perpendicular to the carbon plane. In this case, the $\mathrm{O}-\mathrm{O}$ bond is kept with its binding energy of $92 \mathrm{~kJ} / \mathrm{mol}$ (LDA) and a bond length of $1.45 \AA$, longer than that of a free $\mathrm{O}_{2}$ molecule (1.21 $\mathrm{A}$ ). Because of a small $\mathrm{O}-\mathrm{O}$ bond energy, the top oxygen atom will be easily desorbed at typical etching temperatures and can participate in the oxidation at other active sites. We note that this type of an $\mathrm{O}_{2} *$ precursor state does not exist at the top sites. An oxygen atom is exothermally released from one top site and adsorbed to another top site nearby.

The dissociative adsorption of an $\mathrm{O}_{2}$ molecule at two top sites and two bridge sites gains energy of $-531(-473)$ and -974 $(-830) \mathrm{kJ} / \mathrm{mol}$, respectively. Since the oxide at the bridge site is more stable than at the top site, the CO moiety of the topsite oxide is more liable to be etched away to generate gaseous $\mathrm{CO}$ at an elevated temperature. Note that $\mathrm{CO}$ back-bonds at the top site are already stretched upward and form two single bonds with a bond length of $1.47 \AA$, as shown in Figure $5 \mathrm{~b}$, suggesting that more serious bond stretching of carbon backbonds is involved at the top sites. During this reaction, the energy is increased by $251(154) \mathrm{kJ} / \mathrm{mol}$, but is still lower than that of the initial state by $280(318) \mathrm{kJ} / \mathrm{mol}$. Thus, the reaction path involving exothermic dissociation of $\mathrm{O}_{2}$ molecules and activated $\mathrm{CO}$ desorption from the top sites is the most likely primary etching process, following ER mechanism. Unlike at the top sites, the $\mathrm{CO}$ bond at the bridge site is strongly bound and is very unlikely to be etched away under normal circumstances. Yet, oxygen atoms at bridge sites can be etched away to desorb $\mathrm{CO}_{2}$ by assistance of an energetic $\mathrm{CO}$ molecule formed at other sites. The energy is increased in this process by 87 (58) $\mathrm{kJ} / \mathrm{mol}$, but is still much lower than that of the initial state of an $\mathrm{O}_{2}$ molecule. Thus, $\mathrm{CO}_{2}$ can be formed only by the secondary etching process involving energetic $\mathrm{CO}$ gas. This explains why the $\mathrm{CO}$ production rate is larger than the $\mathrm{CO}_{2}$ production rate under normal oxidative etching conditions of graphite. ${ }^{33-38}$ We note that, when the $\mathrm{CO}$ back-bond at a bridge site exceeds a bond length of $1.6 \AA, \mathrm{CO}_{2}$ is immediately formed. Oxygen atoms, which can easily be released from the $\mathrm{O}_{2}$ * precursor states, can migrate on the surface and adsorb on the top and bridge sites at vacancies or react with existing oxides.

4.3. Oxidation of Multilayer Vacancy. To examine the adsorption of $\mathrm{O}_{2}$ molecules at multilayer vacancies, we have generated two monovacancies in two adjacent carbon layers, which provides the simplest model of a double-layer vacancy. We have tested various initial positions of intercalated $\mathrm{O}_{2}$ molecule near this vacancy, including the geometry with a $\mathrm{O}-\mathrm{O}$ molecular axis across two carbon planes. In all cases, $\mathrm{O}_{2}$ dissociates to top and bridge sites, respectively, on the two layers, producing the oxide species analogous to the case of monolayer vacancy (Figure 5a). This behavior is reasonable because, if one considers the reaction energetics only, a multilayer process should not be much different from a monolayer process due to negligible energy perturbations caused by the neighbor graphite sheets. The absence of stable intercalated molecular $\mathrm{O}_{2}$ and the similarity in the intermediates for monolayer and multilayer oxidation suggest that the multilayer reaction can simply be interpreted in terms of the monolayer etching mechanism.

A difference in the multilayer etching, however, is that $\mathrm{O}_{2}$ molecules can be supplied to the vacancies at sublayers. The $\mathrm{O}_{2}$ reaction forms oxides at the top and bridge sites as well as the $\mathrm{O}_{2} *$ precursor species in the sublayer. The top-site oxide can be desorbed as $\mathrm{CO}$ gas, similar to the case of monolayer etching. The $\mathrm{CO}$ desorbed from the sublayer migrates out, and in this process it can react with oxygen atoms in the upper layers.

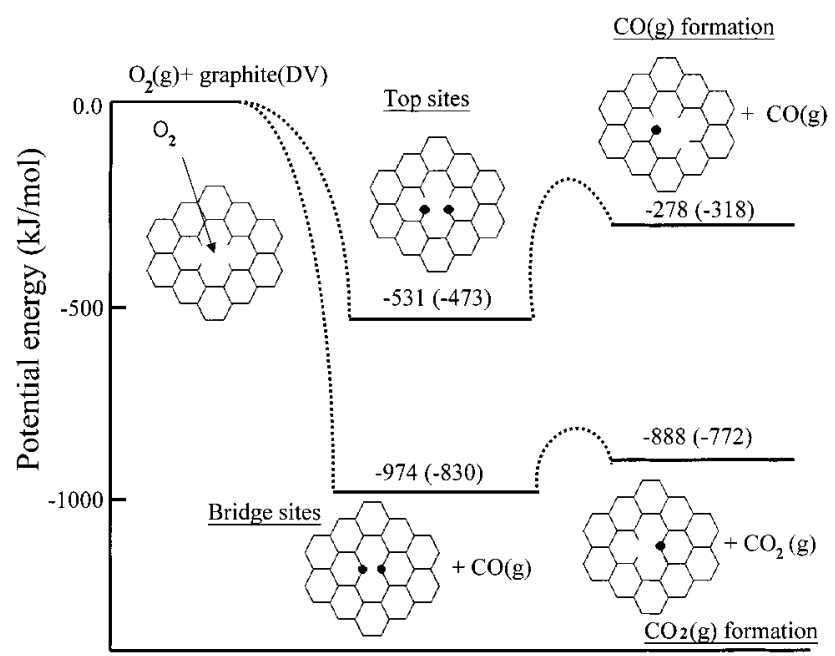

Figure 6. The reaction energy diagram for $\mathrm{O}_{2}$ adsorption at a divacancy and desorption processes to produce $\mathrm{CO}$ and $\mathrm{CO}_{2}$ gases. The top site oxygen is desorbed as $\mathrm{CO} . \mathrm{CO}_{2}$ is formed by reaction of $\mathrm{CO}$ gas with the bridge site oxygen.

This secondary reaction allows one to remove the oxygen atoms not only from the top site but also from the more stable bridge site. The gaseous $\mathrm{O}$ atoms released from the $\mathrm{O}_{2}{ }^{*}$ precursors can also induce further reactions with the oxide species. The most viable products of these secondary reactions are $\mathrm{CO}_{2}$ gas, as demonstrated in the reaction energy diagram of Figure 6.

\section{Discussion}

5.1. Mechanism of Monolayer Oxidation. According to the previous investigations, ${ }^{24-26} \mathrm{Ar}^{+}$ion impact creates two types of graphite defects: carbon vacancy and Ar interstitial. The vacancy can be created by displacement of surface atom(s) by the $\mathrm{Ar}^{+}$- surface collision, and the interstitial by trapping of an incident $\mathrm{Ar}^{+}$beneath a carbon plane after ion surface penetration and neutralization. Both of these defects are converted to pits by the $\mathrm{O}_{2}$ etching reaction, but with different efficiencies. We have previously observed ${ }^{27}$ that $93 \%$ of the vacancies are converted to pits, while the conversion efficiency of the interstitials is only $14 \%$ when they are created by $100 \mathrm{eV} \mathrm{Ar}^{+}$ impact. This result suggests that in the present experimental conditions a majority of the pits are originated from the vacancies. In the following discussion, we explain the mechanism of defect oxidation as if the oxidation starts from vacancies. Though the initial oxidation mechanism of an interstitial leaves an interesting question, i.e., how the surface carbon layer deformed by an Ar interstial is attacked by an $\mathrm{O}_{2}$ molecule; once a defect hole is created at the top layer by the initial etching of an interstitial, then the rest of the etching process should be identical to the case of vacancy oxidation.

STM observation reveals two types of monolayer vacancy etching, resulting in isotropic and anisotropic serpentine structures. The isotropic etching has been known since the early studies involving natural defects. ${ }^{5-20}$ However, the serpentine etching of an impurity-free graphite has not been reported previously. The DF calculation results of section 4.2 provide a reasonable picture for the different etching patterns. In monolayer etching, with a continuous supply of $\mathrm{O}_{2}$ molecules to vacancies, both the top and bridge $\mathrm{O}$ species can be formed with no activation barrier. The $\mathrm{CO}$ moiety at the top site is relatively weakly bound [251 (154) kJ/mol] and can be desorbed by a thermally activated process, while the bridge oxide species is unlikely to be etched away due to a stronger bond. Once a $\mathrm{CO}$ is desorbed from the top site, two dangling bonds are newly 


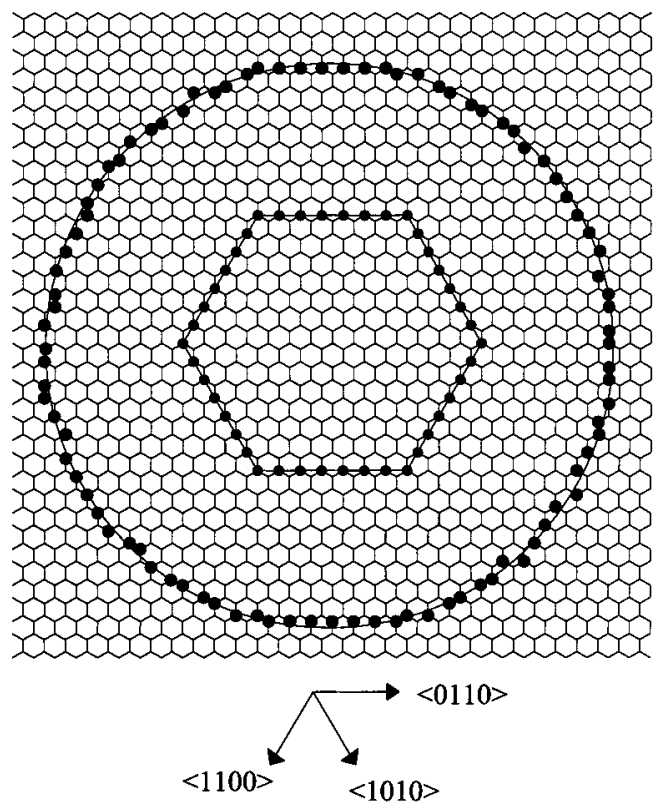

Figure 7. Schematic representation for the structure of a hexagonal pit and a larger circular pit. The solid dots represent carbon dangling bonds. The large circle and the hexagon are drawn to depict pit boundaries.

created, again favoring the formation of either two top-site oxides or an $\mathrm{O}_{2}$ * precursor state. If the two oxides are formed, etching will further continue in the same direction, leading to directional etching or channel formation as shown in Figure 1e. If an $\mathrm{O}_{2} *$ precursor state is formed, etching will not proceed to that direction. Still, etching may be continued in other directions where reactive top sites exist. This causes a bending of the etched channel or a serpentine structure.

At the initial stage of the etching reaction or when a pit diameter is very small $(<30 \mathrm{~nm})$, STM images often show hexagonally shaped pits. Removal of the top-site oxides and concurrent generation of new dangling bonds, described in the previous paragraph, proceeds preferentially along the graphite lattice directions, i.e., $\langle 1100\rangle,\langle 1010\rangle$, and $\langle 0110\rangle$ directions. Such directional etching, when occurs to all three directions with about equal probability, will generate a hexagonal pit, as illustrated in Figure 7. Upon substantial growth of a pit size, however, the number of carbons at the hexagonal sides becomes far larger than the six corner atoms, and thus it becomes more probable to etch away the side carbons. The side atoms are removed by random attack of reactive species, and this will result in a curved boundary. Statistical compromise between the directional etching and the random etching will give rise to an approximately circular shape for an etched pit (Figure 7).

5.2. Mechanism of Multilayer Oxidation. The STM results show several unique features for the formation of multilayer pits. The multilayer etching occurs simultaneously for the top and inner carbon layers at the pit boundary, always producing nearly circular pits. The multilayer etching occurs several times faster than the monolayer etching. The fast etch speed comes from a large pre-exponential factor. These observations suggest that a different type of etching process may be involved in the multilayer etching. The DF calculation, however, shows that $\mathrm{O}_{2}$ reaction gives rise to similar initial chemisorption geometries at both monolayer and multilayer vacancies. These STM and DF results might seem contradictory with each other. In the following, we address this issue.

A distinct difference exists between the monolayer and multilayer etching in three-dimensional reaction environment.
Consider $\mathrm{O}_{2}$ chemisorption at a multilayer vacancy described in section 4.3. The chemisorption can produce the oxides or $\mathrm{O}_{2}{ }^{*}$ species in the sublayer, which, at elevated temperature, liberates gaseous $\mathrm{CO}$ or $\mathrm{O}$ atoms, respectively. These reactive species, when created from the inner carbon layers of a multilayer pit, will have a higher probability for encountering and reacting with the oxide species present at the upper layers of an etched boundary. Increased reactivity of the $\mathrm{CO}$ or $\mathrm{O}$ species is also assisted by their relatively longer stay time inside a deep well, either in a gaseous state or weakly physisorbed state. The secondary reactions of $\mathrm{CO}$ and $\mathrm{O}$ allow one to remove the oxides not only from the top sites but also from the more stable bridge sites by forming $\mathrm{CO}_{2}$ gas. Since this occurs randomly and at an increased efficiency for the multilayer etching, anisotropic etching is relatively suppressed and a circular etching pattern should appear. This mechanistic interpretation is consistent with the kinetic parameters obtained by the STM study: the higher $A$ factor (higher collision rate of reactant) for multilayer etching and the similar $E_{\mathrm{a}}$ values (similar energies for the transition states) for both mono- and multilayer etching.

According to the mechanism proposed above, the ratio of $\mathrm{CO}_{2} / \mathrm{CO}$ in the desorption products will be larger for multilayer etching than for monolayer etching. Several previous studies show that it is indeed the case. ${ }^{35-38}$ Thermal oxidation of a graphite sample under $10^{-5}$ Torr of $\mathrm{O}_{2}$ gas after sputtering by $5 \mathrm{keV} \mathrm{Ar}{ }^{+}$ions produced $\mathrm{CO}_{2}$ product in higher abundancy than $\mathrm{CO} .{ }^{38}$ The high-energy ion sputtering must have produced mostly multilayer defects on the surface. ${ }^{39}$ On the other hand, the thermal desorption studies for oxidation of graphite monolayer formed on metal surfaces ${ }^{35-37}$ revealed that $\mathrm{CO}$ is the major desorption product, while $\mathrm{CO}_{2}$ is produced only by about $1 \%$.

\section{Summary and Conclusions}

In this work, we have carried out systematic investigations for the oxidative etching of monolayer and multilayer defects at a graphite surface by using STM and DF calculation. We summarize the important findings of this study as follows.

(i) Thermal oxidation of a defect leads to pit formation by layer-by-layer etching of the carbon layer. Oxidation of monolayer defects produces round-shaped pits and less frequently, anisotropically etched pits. Multilayer defect oxidation always produces circular pits. The etching occurs faster at multilayer carbon edges than at monolayer edges, via simultaneous removal of the top and inner-layer carbons. The etch rate increases by $2-3$ times as the pit depth is increased by one carbon layer up to three.

(ii) A temperature dependence study of the etch rate shows an Arrhenius behavior, from which the associated kinetic parameters are deduced. The activation energies for the etching reactions are $96 \pm 8 \mathrm{~kJ} / \mathrm{mol}$ (monolayer), $109 \pm 5 \mathrm{~kJ} / \mathrm{mol}$ (double-layer), and about $130 \mathrm{~kJ} / \mathrm{mol}$ (triple-layer). The corresponding $A$ factors are $10^{4}-10^{5} \mathrm{~s}^{-1}$ (monolayer), $10^{6} \mathrm{~s}^{-1}$ (double-layer), and $10^{8} \mathrm{~s}^{-1}$ (triple-layer). These numbers reveal that a fast etch rate of a multilayer pit comes from a large $A$ factor.

(iii) DF calculation is performed to identify the adsorption structures of $\mathrm{O}_{2}$ at vacancy sites; atomic $\mathrm{O}$ at the top and bridge sites and molecular $\mathrm{O}_{2} *$ precursor state at the bridge site. These species are commonly produced in both monolayer and multilayer oxidation.

(iv) The reaction pathways are proposed for monolayer and multilayer etching. Monolayer etching involves $\mathrm{O}_{2}$ chemisorp- 
tion at the carbon dangling-bond sites followed by thermally activated desorption of $\mathrm{CO}$ gas. In addition to this $\mathrm{CO}$ production channel, multilayer etching can also proceed via secondary reactions between the surface oxides and the energetic $\mathrm{CO}$ and $\mathrm{O}$ atoms generated from the inner carbon layers of a pit, producing $\mathrm{CO}_{2}$ gas. A round shaped pit is favorably formed by statistical etching of the edge carbon atoms, whereas an anisotropic structure results from directional etching.

Acknowledgment. This work was supported by the Creative Research Initiatives Project of the Ministry of Science and Technology (H.K.) and by Korea Science and Engineering Foundation through the Semiconductor Physics Research Center (Y.H.L.).

\section{References and Notes}

(1) Thomas, J. M. In Chemistry and Physics of Carbon; Walker, P. L., Jr., Ed.; Marcel Dekker: New York, 1965; Vol. 1, pp 121-203.

(2) Hennig, G. R. In Chemistry and Physics of Carbon; Walker, P. L., Jr., Ed.; Marcel Dekker: New York, 1966; Vol. 2, pp 1-50.

(3) Mckee, D. W. In Chemistry and Physics of Carbon; Walker, P. L., Jr., Ed.; Marcel Dekker: New York, 1981; Vol. 16, pp 1-112.

(4) Yang, R. T. In Chemistry and Physics of Carbon; Walker, P. L., Jr., Thrower, P. A., Eds.; Marcel Dekker: New York, 1984; Vol. 19, pp $163-210$ 174

(5) Evans, E. L.; Griffiths, R. J. M.; Thomas, J. M. Science 1971, 171,

(6) Wong, C.; Yang, R. T. Carbon 1982, 20, 2539.

(7) Wong, C.; Yang, R. T. J. Chem. Phys. 1983, 78, 3325.

(8) Hennig, G. R. Science 1965, 147, 733.

(9) Hennig, G. R. J. Chem. Phys. 1964, 40, 2877.

(10) Yang, R. T.; Wong, C. Science 1981, 214, 437.

(11) Yang, R. T.; Wong, C. J. Chem. Phys. 1981, 75, 4471.

(12) Yang, R. T.; Wong, C. J. Catal. 1983, 82, 245.

(13) Goethel, P. J.; Yang, R. T. J. Catal. 1989, 119, 201.
(14) Barber, M.; Evans, E. L.; Thomas, J. M. Chem. Phys. Lett. 1973, $18,423$.

(15) Tracz, A.; Wegner, G.; Rabe, J. P. Langmuir 1993, 9, 3033.

(16) (a) Chang, H.; Bard, A. J. J. Am. Chem. Soc. 1990, 112, 4598. (b)

Chang, H.; Bard, A. J. J. Am. Chem. Soc. 1991, 113, 5588.

(17) Tracz, A.; Kalachev, A. A.; Wegner, G.; Rabe, J. P. Langmuir 1995, $11,2840$.

(18) Chu, X.; Schmidt, L. D. Surf. Sci. 1992, 268, 325.

(19) Chu, X.; Schmidt, L. D. Carbon 1991, 29, 1251.

(20) Henschke, B.; Schubert, H.; Blöcker, J.; Atamny, F.; Schlögl, R. Thermochim. Acta 1994, 234, 53.

(21) Patrick, D. L.; Cee, V. J.; Beebe, T. P. Jr. Science 1994, 265, 231.

(22) Shedd, G. M.; Russell, P. E. J. Vac. Sci. Technol. A 1991, 9, 1261.

(23) Marton, D.; Bu, H.; Boyd, K. J.; Todorov, S. S.; Al-Bayati, A. H.; Rabalais, J. W. Surf. Sci. 1995, 326, L489.

(24) Hahn, J. R.; Kang, H. Phys. Rev. B 1999, 60, 6007.

(25) Kang, H.; Park, K. H.; Kim, C.; Shim, S. B.; Kim, S.; Moon, D. W. Nucl. Instrum. Methods in Phys. Res. B 1992, 67, 312.

(26) Hahn, J. R.; Kang, H.; Song, S.; Jeon, I. C. Phys. Rev. B 1996, 53 , R1725.

(27) Hahn, J. R.; Kang, H. J. Vac. Sci. Technol. A 1999, 17, 1606.

(28) Lee, S. M.; Lee, Y. H.; Hwang, Y. G.; Hahn, J. R.; Kang, H. Phys. Rev. Lett. 1999, 82, 217

(29) Hahn, J. R. Surf. Sci. 1999, 423, L216.

(30) Wang, X.-S.; Huang, C.; Bressler-Hill, V.; Maboudian, R.; Weinberg, W. H. J. Vac. Sci. Technol. A 1993, 11, 2860.

(31) Park, K. H.; Kim, B. C.; Kang, H. J. Chem. Phys. 1992, 97, 2742.

(32) DMol3 is a registered software product of Molecular Simulations, Inc. Delley, B. J. Chem. Phys. 1990, 92, 508.

(33) Perdew, J. P.; Wang, Y. Phys. Rev. B 1992, 45, 13244.

(34) Becke, A. D. J. Chem. Phys. 1988, 88, 2547.

(35) Yang, Y. W.; Hrbek, J. J. Phys. Chem. 1995, 99, 3229.

(36) Lauderback, L. L.; Delgass, W. N. Surf. Sci. 1986, 172, 715.

(37) Shincho, E.; Egawa, C.; Naito, S.; Tamaru, K. Surf. Sci. 1985, 149 ,

(38) Atamny, F.; Blöcker, J.; Henschke, B.; Schlögl, R.; Schedel-Niedrig, Th.; Keil, M.; Bradshaw, A. M. J. Phys. Chem. 1992, 96, 4522.

(39) Bräuchle, G.; Richard-Schneider, S.; Illig, D.; Beck, R. D. Schreiber, H.; Kappes, M. M. Nucl. Instrum. Methods in Phys. Res. B 1996, 112,105 . 